\title{
Effect of biochar application at a trace-elements polluted area on soil carbon stability
}

\author{
$\underline{\text { Paloma Campos }}^{1}$, Ana Z. Miller ${ }^{2}$, Heike Knicker ${ }^{1}$, Águeda Sánchez-Martín ${ }^{1}$, \\ Elena Fernández-Boy ${ }^{3}$, José María De la Rosa ${ }^{1}$ \\ 1. Instituto de Recursos Naturales y Agrobiología de Sevilla (IRNAS-CSIC), Av. Reina Mercedes 10, 41012, Sevilla, Spain \\ 3. Facultad de Química, Universidad de Sevilla. Profesor García González St. 41012, Seville, Spain
}

2. HERCULES Laboratory, University of Évora, Évora, Portugal.

pcamposdm@irnas.csic.es 


\section{What is biochar?}

pcampos@irnas.csic.es

Biochar (BC) is the carbonaceous solid residue produced through the thermochemical conversion (pyrolysis) of biomass under low $\mathrm{O}_{2}$ atmosphere.

\section{Biomass}

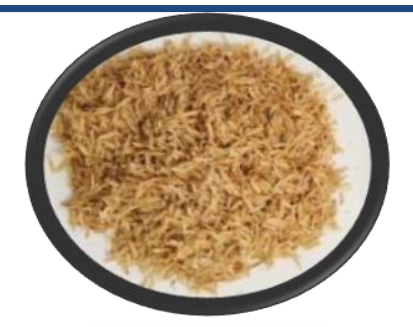

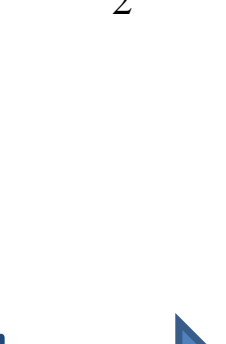

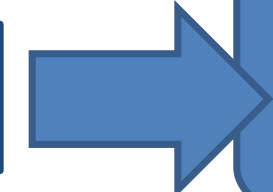

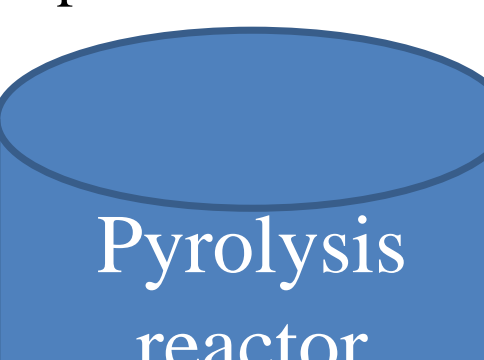

\section{reactor}
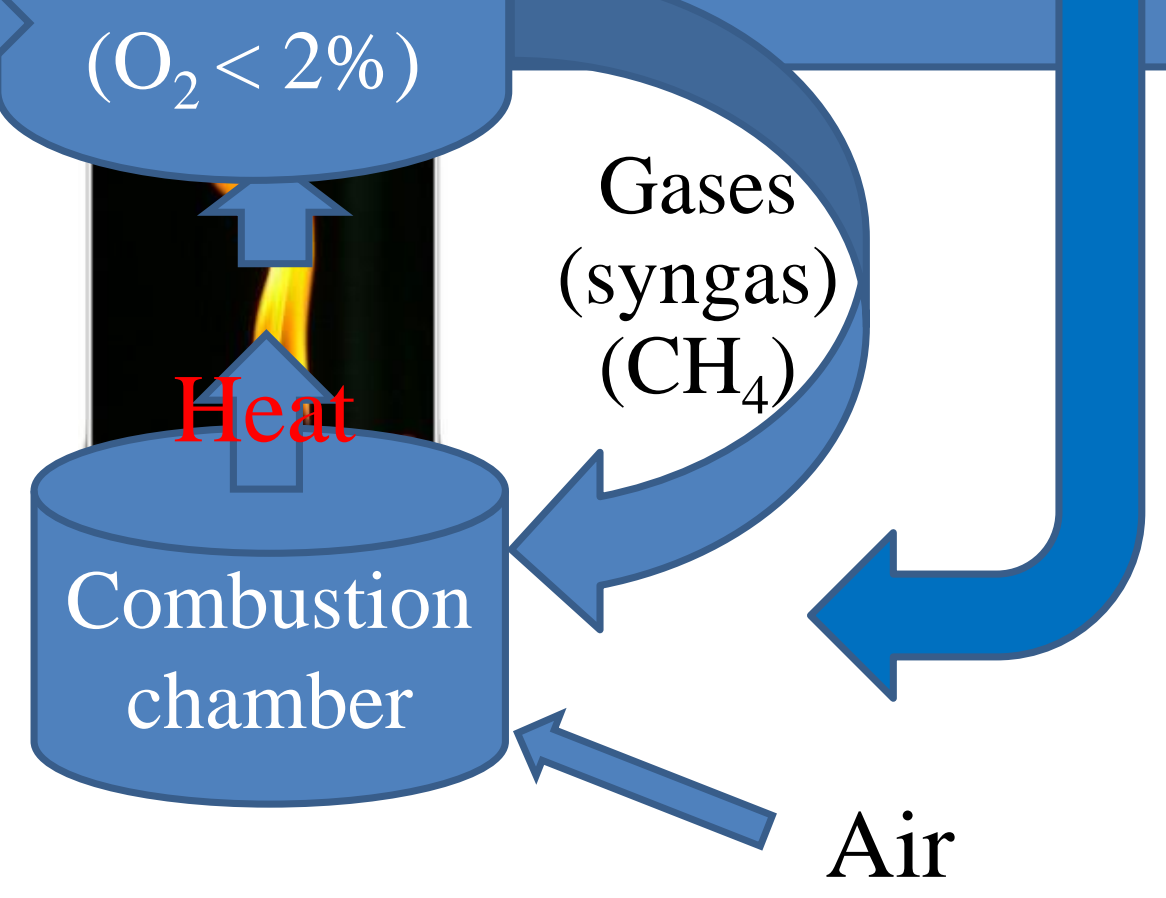

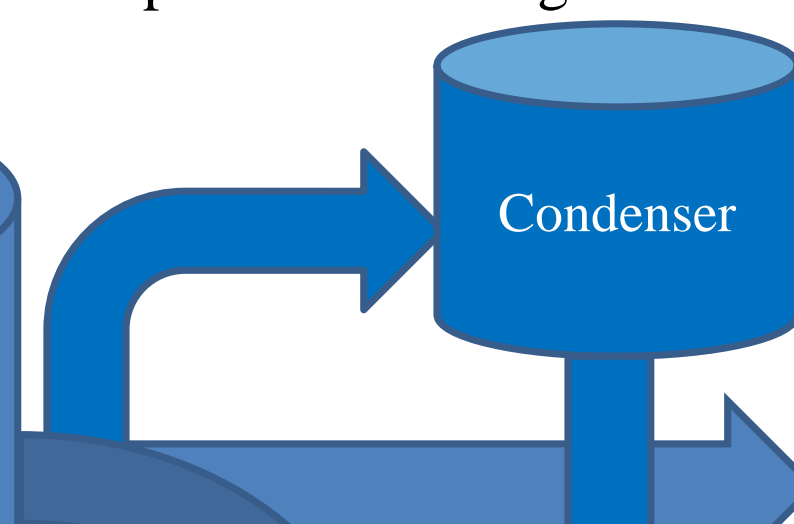

BIOCHAR

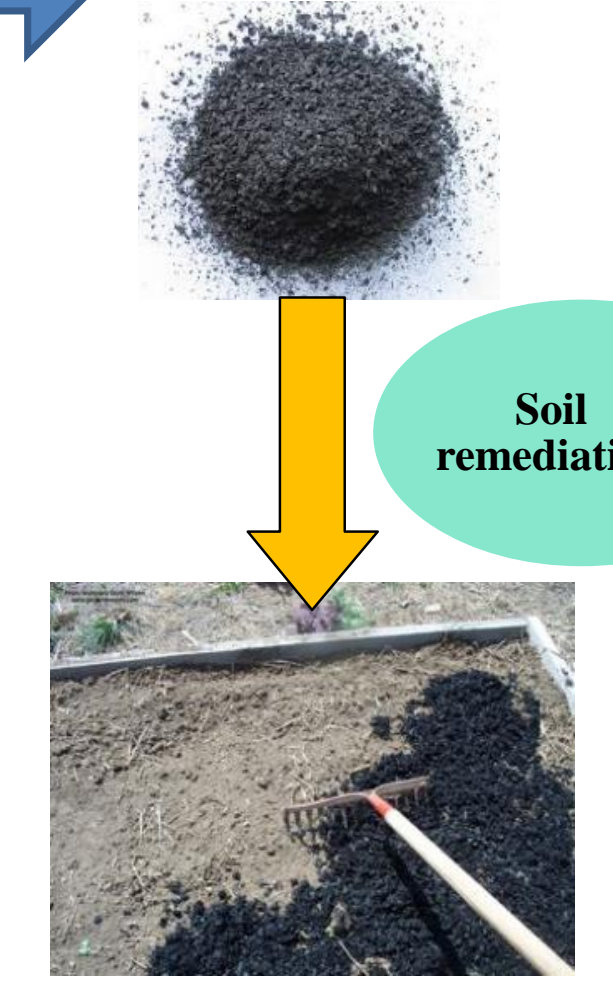

Soil (c) (1) 


\section{Biochar applications}

pcampos@irnas.csic.es

Biochar properties:

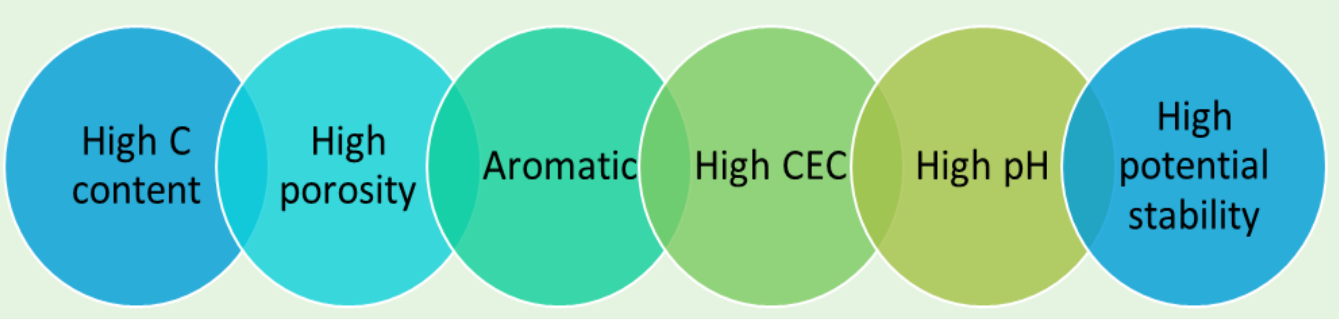

Different applications:

- Improvement of soil fertility, plant growth

- Reduction of soil nutrient leaching

- Mitigation of green house gases

- C sequestration

- Soil remediation

Biochar potential to immobilize trace elements

\section{Direct mechanisms}

\section{Chemisorption}

- Adsorption in oxygenated functional groups (release $\mathrm{H}^{+}$) - Cationic exchange (Na. Ca, S. K. Mg)

Physisorption

- Electrostatic attraction ( $\pi$ electrons)

\section{Precipitation}

- Mineral fraction / biochar ash

\section{Indirect mechanisms}

\begin{tabular}{|c|}
\hline Changes in $\mathrm{pH}$ \\
\hline Organic matter and soluble $\mathrm{C}$ \\
\hline Priming effect and Doc \\
\hline Availability of $\mathrm{P}$ \\
\hline Competition with As \\
\hline Redox \\
\hline
\end{tabular}




\section{Biochar stability?}

pcampos@irnas.csic.es

\section{Stability of biochars under debate}

MRT $_{\text {biochar }}>1000$ years, when $\mathrm{H} / \mathrm{C}_{\text {org }}<0.4$ $\mathrm{MRT}_{\text {biochar }}>500, \quad$ when $0.4<\mathrm{H} / \mathrm{C}_{\text {org }}<0.7$

(Lehmann and Joseph, 2015)

\begin{tabular}{|l|l|l|}
\hline Reference & $\begin{array}{l}\text { Scale of } \\
\text { estimated } \\
\text { MRT }\end{array}$ & MTT (years) \\
\hline Masiello and Druffel (1998) & Millennial & $2400-13900$ \\
\hline Cheng et al. (2006) & Millennial & 1000 \\
\hline Kuzyakov et al. (2009) & Millennial & 2000 \\
\hline Novak et a. (2010) & Millennial & $1400-51000$ \\
\hline Zimmerman (2010) & $\begin{array}{l}\text { Centennial to } \\
\text { millennial }\end{array}$ & $100-100000$ \\
\hline
\end{tabular}

De la Rosa et al. (2018)

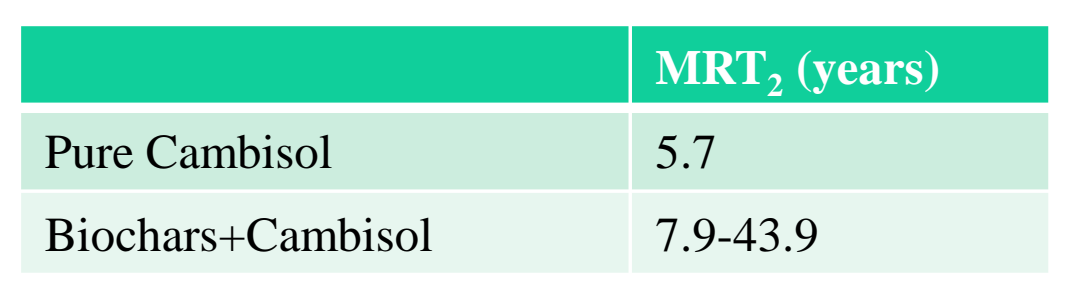

Dos Anjos Leal et al. (2019)

\begin{tabular}{|l|l|}
\hline & MRT $_{2}$ (years) \\
\hline Pure Cambisol & 15.7 \\
\hline Biochar+Cambisol & 17.4 \\
\hline Pure biochar & 87 \\
\hline
\end{tabular}

Main objetive: Determine the effects of biochar on C stability in trace element polluted soils. 


\section{Respiration experiment $-\mathrm{C}$ stability ${ }^{\text {camposeimascscices }}$}
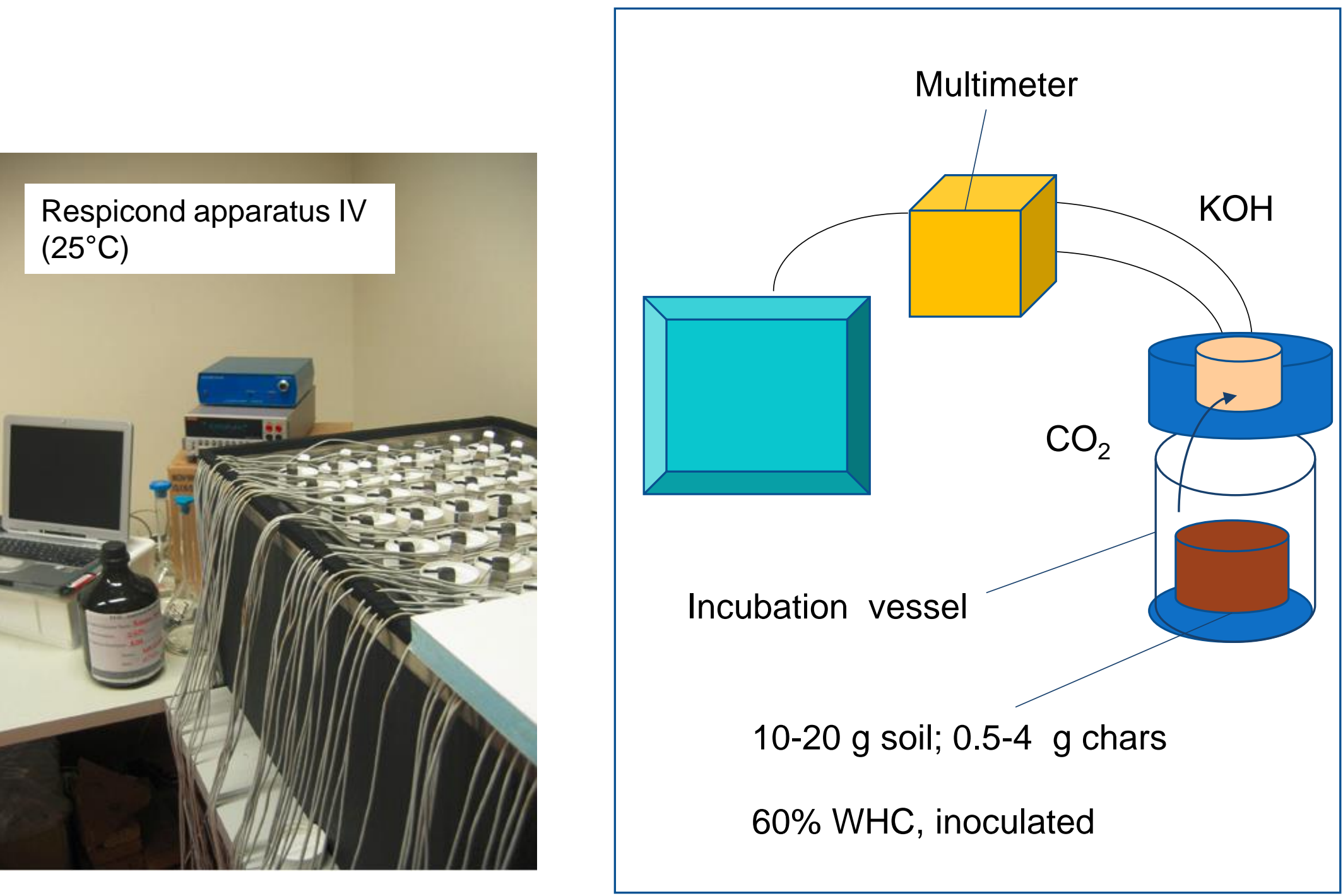

(9) $(1)$ 


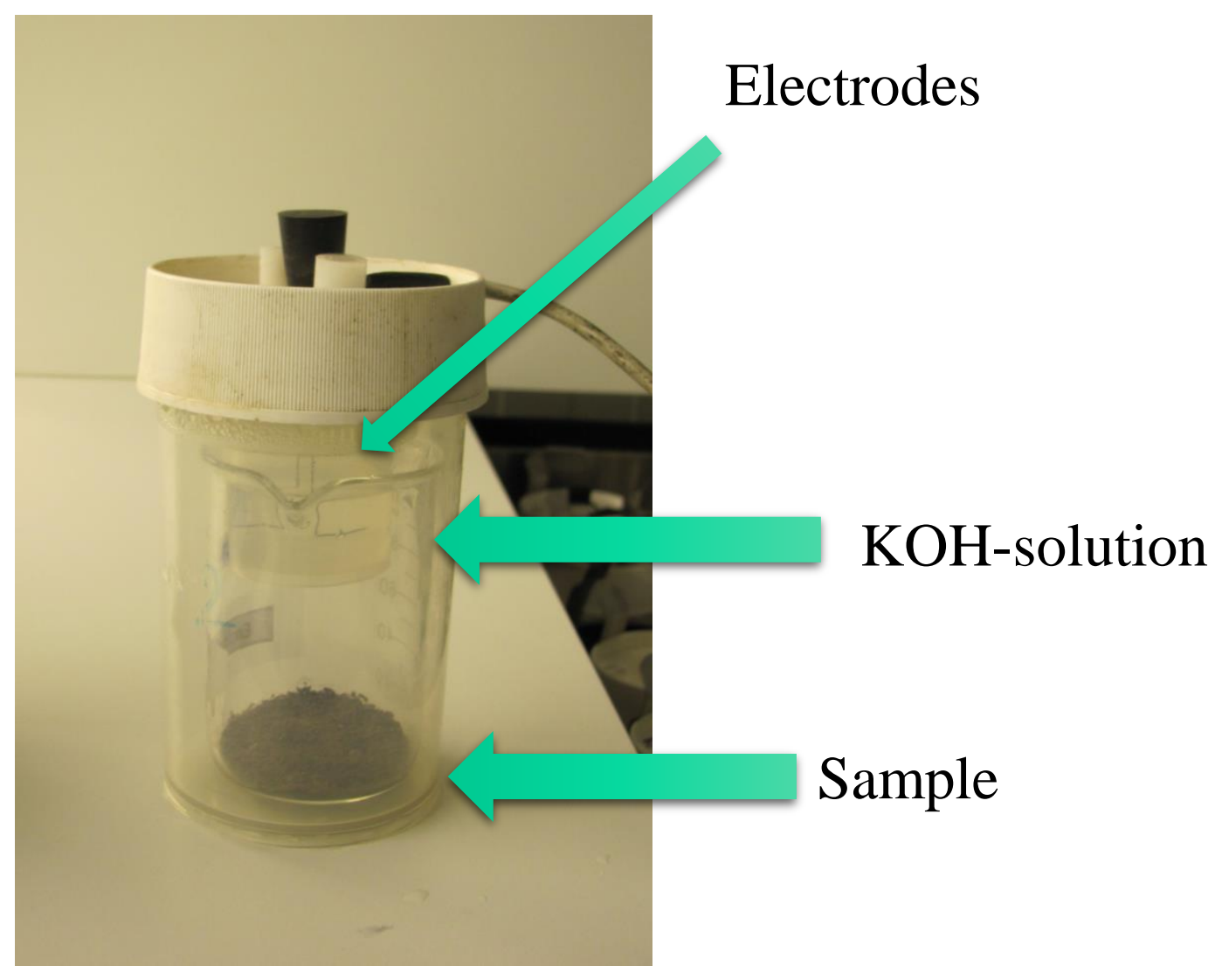

1. $\mathrm{CO}_{2}$ released by the sample is absorbed in the $\mathrm{KOH}$-solution and forms carbonate, which decrease the conductivity.

2. This change is conductivity is measured by the respirometer.

3. From these values can be calculated:

- Cumulative carbon loss

- Degradation rates

- Mean residence time. 


\section{Respiration experiment $-\mathrm{C}$ stability}

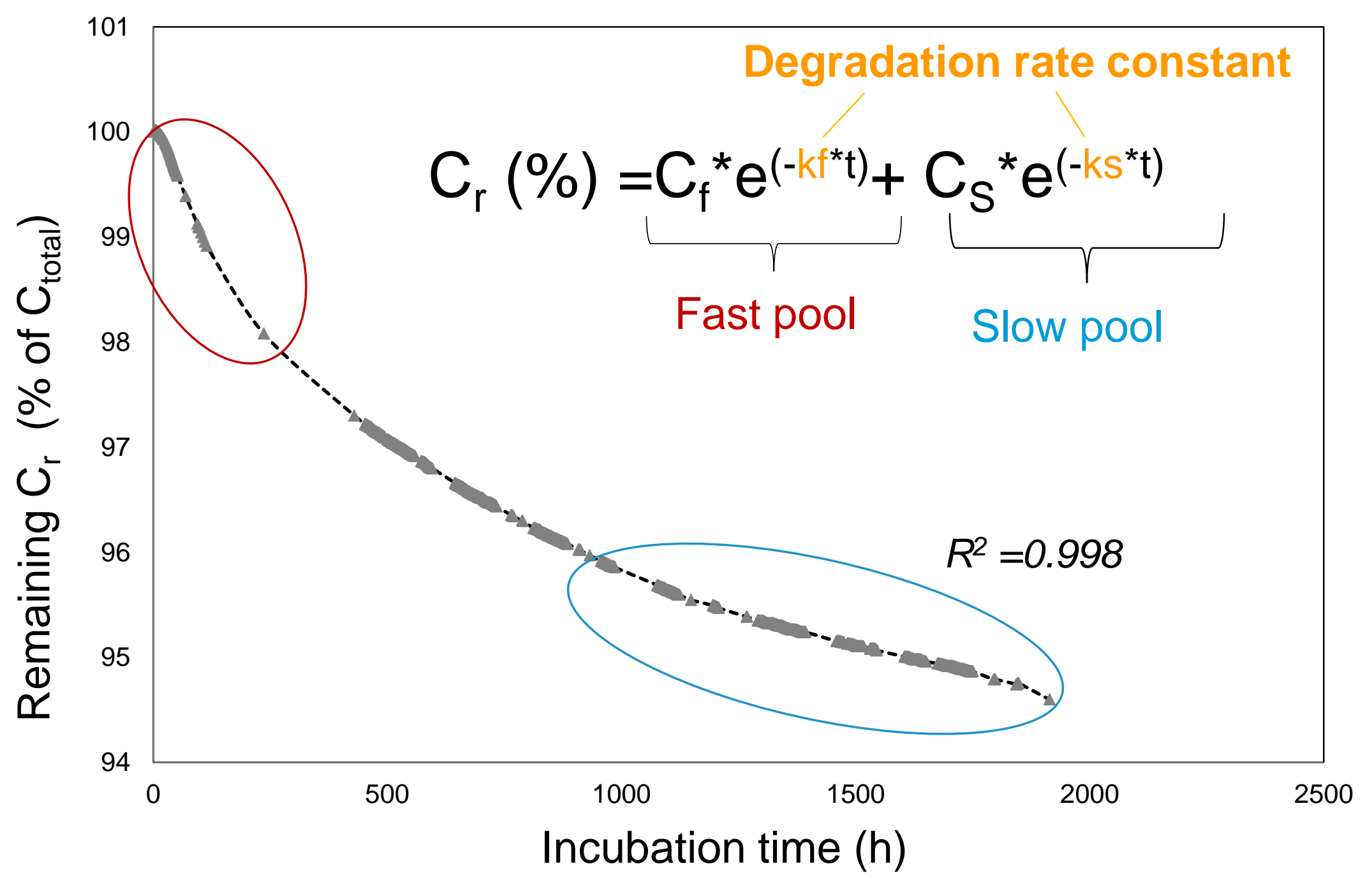

(c) (1) 


\section{Materials: soils}

pcampos@irnas.csic.es

Green Corridor of Guadiamar (25 km north Seville)

2 soil (Typic Xerofluvent):

MPS: Moderately Polluted Soil

HPS: High Polluted Soil

\begin{tabular}{|cccccc|}
\hline & WHC (\%) & Bulk density $(\mathrm{g} / \mathbf{m l})$ & $\mathbf{p H}\left(\mathbf{C a C l}_{2}\right)$ & $\mathbf{E C ~}(\boldsymbol{\mu S} / \mathbf{c m})$ & $\% \mathbf{C}$ \\
\hline HPS & 32.73 & 1.1 & 3.62 & 3610 & 0.8 \\
\hline MPS & 51.45 & 1.2 & 6.47 & 1809 & 1.7 \\
\hline
\end{tabular}

The Aznalcóllar Mine Accident

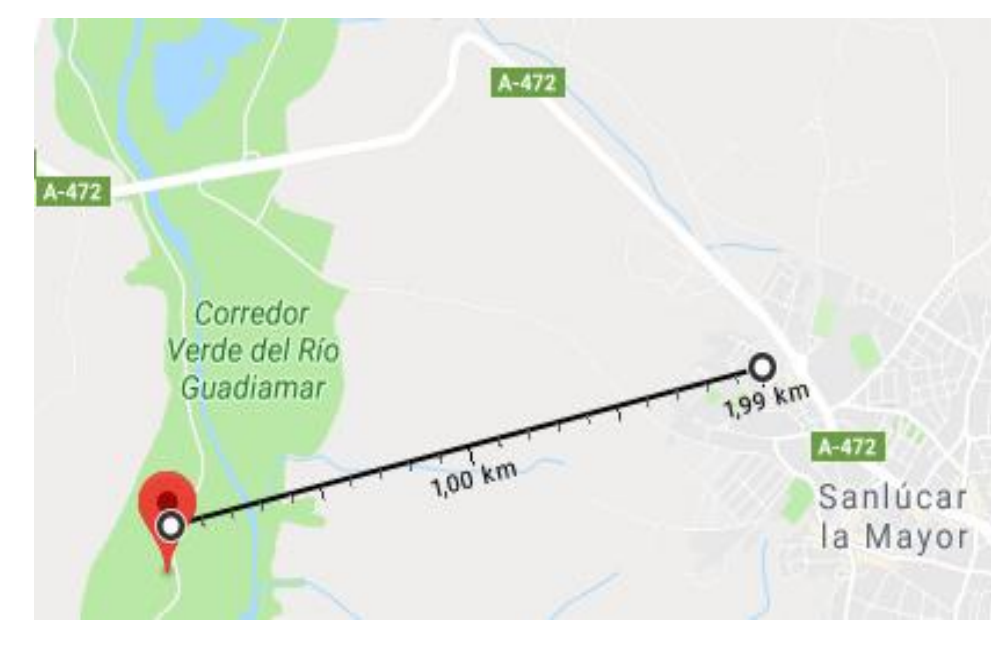

HPS - Highly Polluted Soil

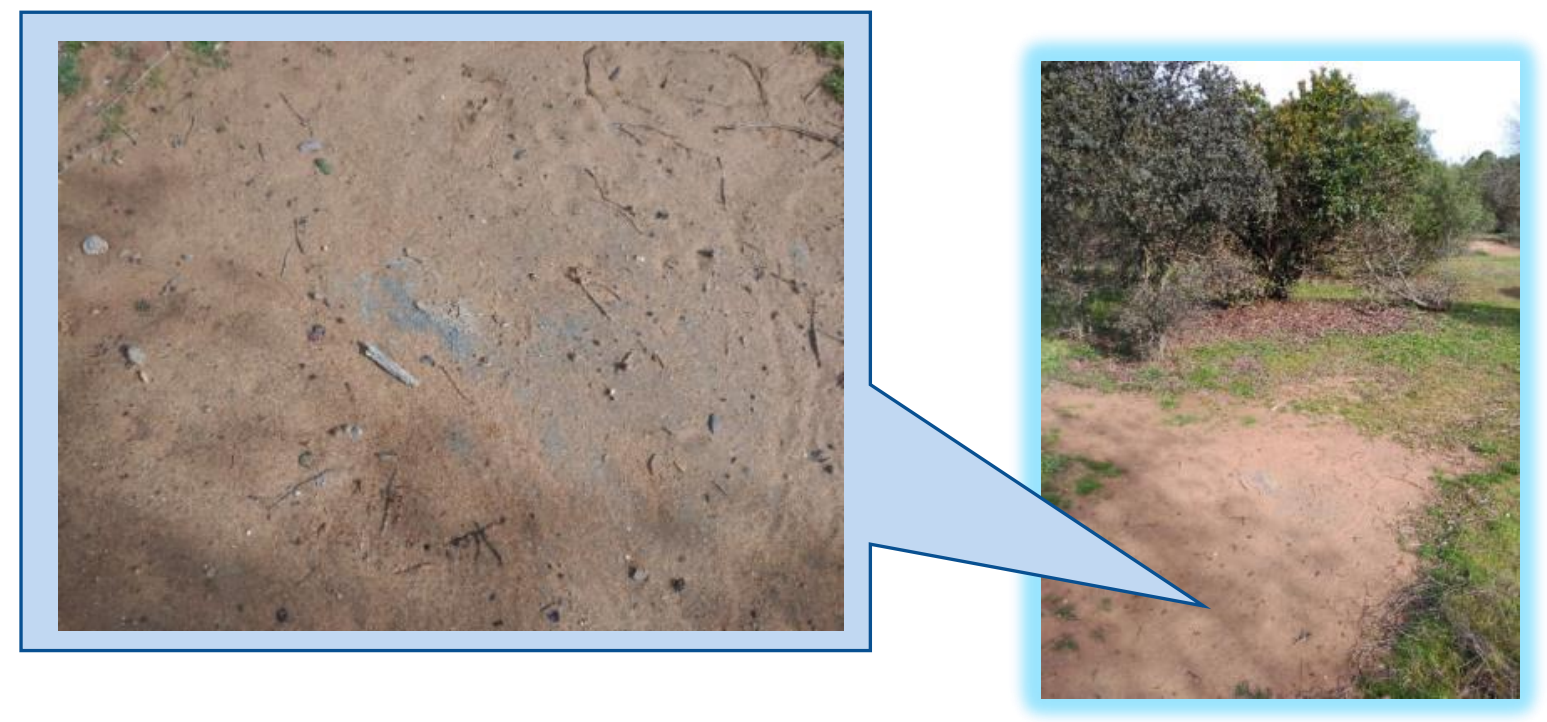




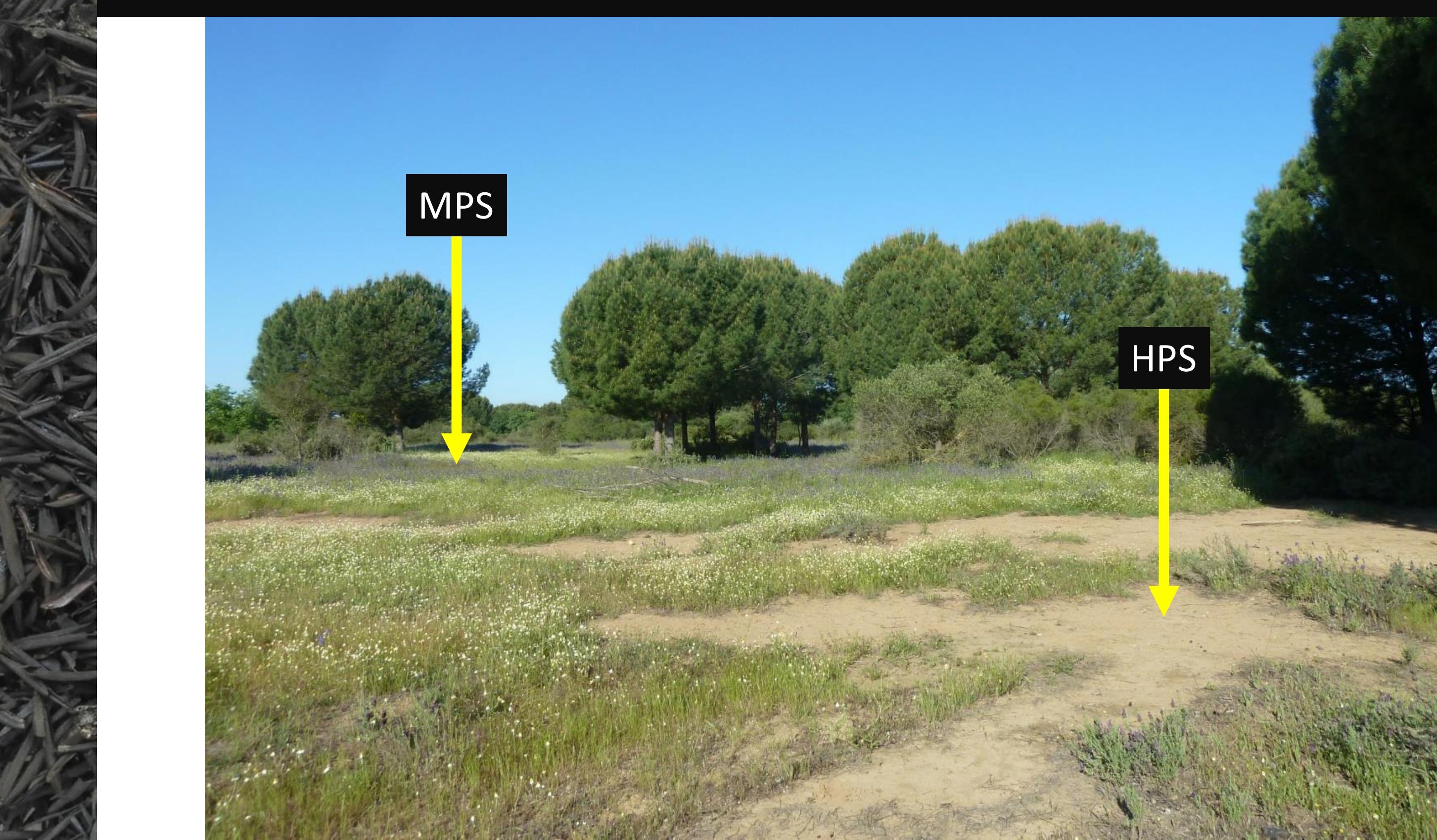

(c) 0 


\section{The Aznalcóllar Mine Accident}

Location:

-Iberian Pyrite Belt (SW Spain-Portugal)

-Aznalcóllar (30 km from Seville)

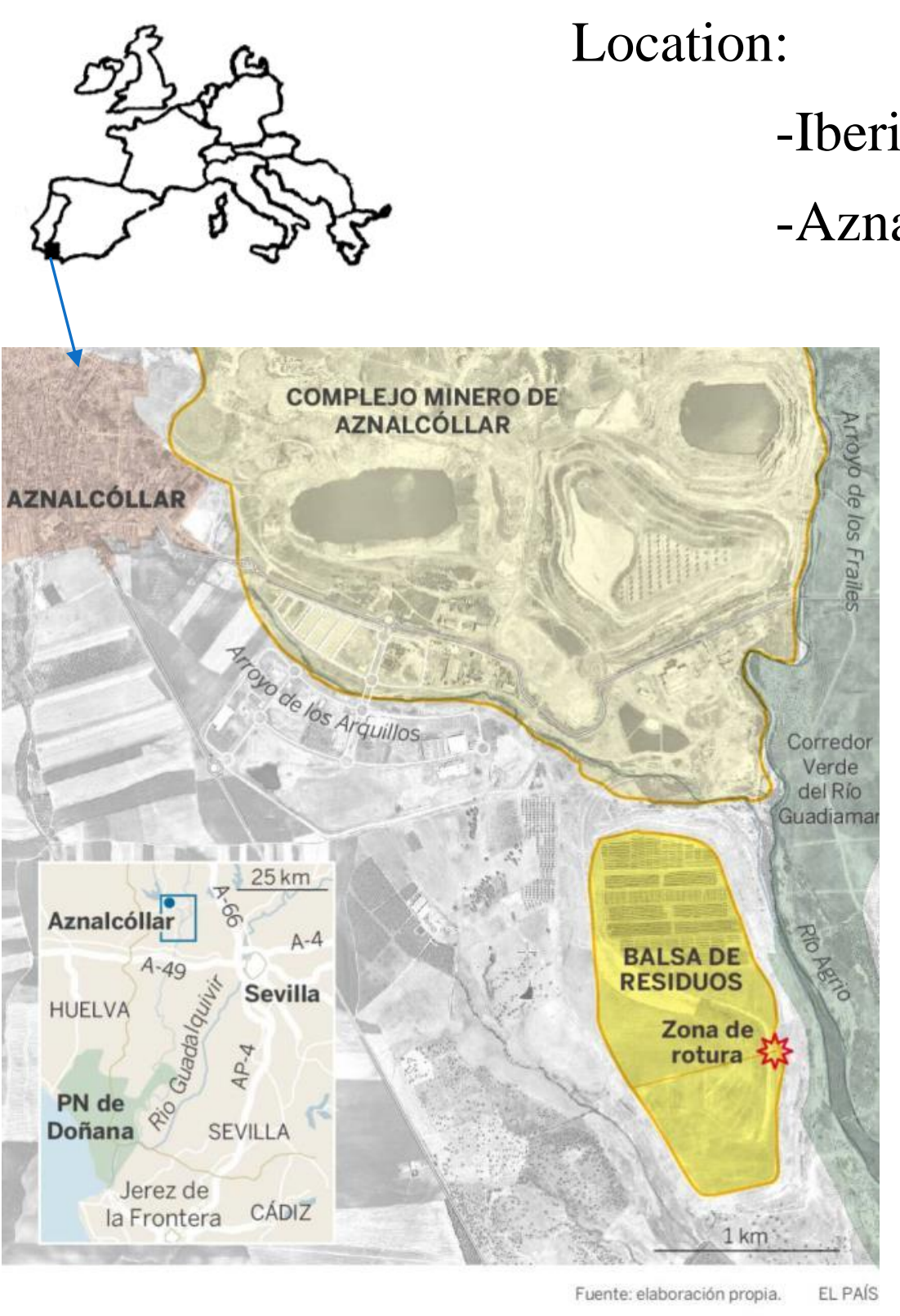

$25^{\text {th }}$ April 1998 (3:30 am)

- 4 millions $\mathrm{m}^{3}$ acidic water

- 2 millions $\mathrm{m}^{3}$ toxic mud

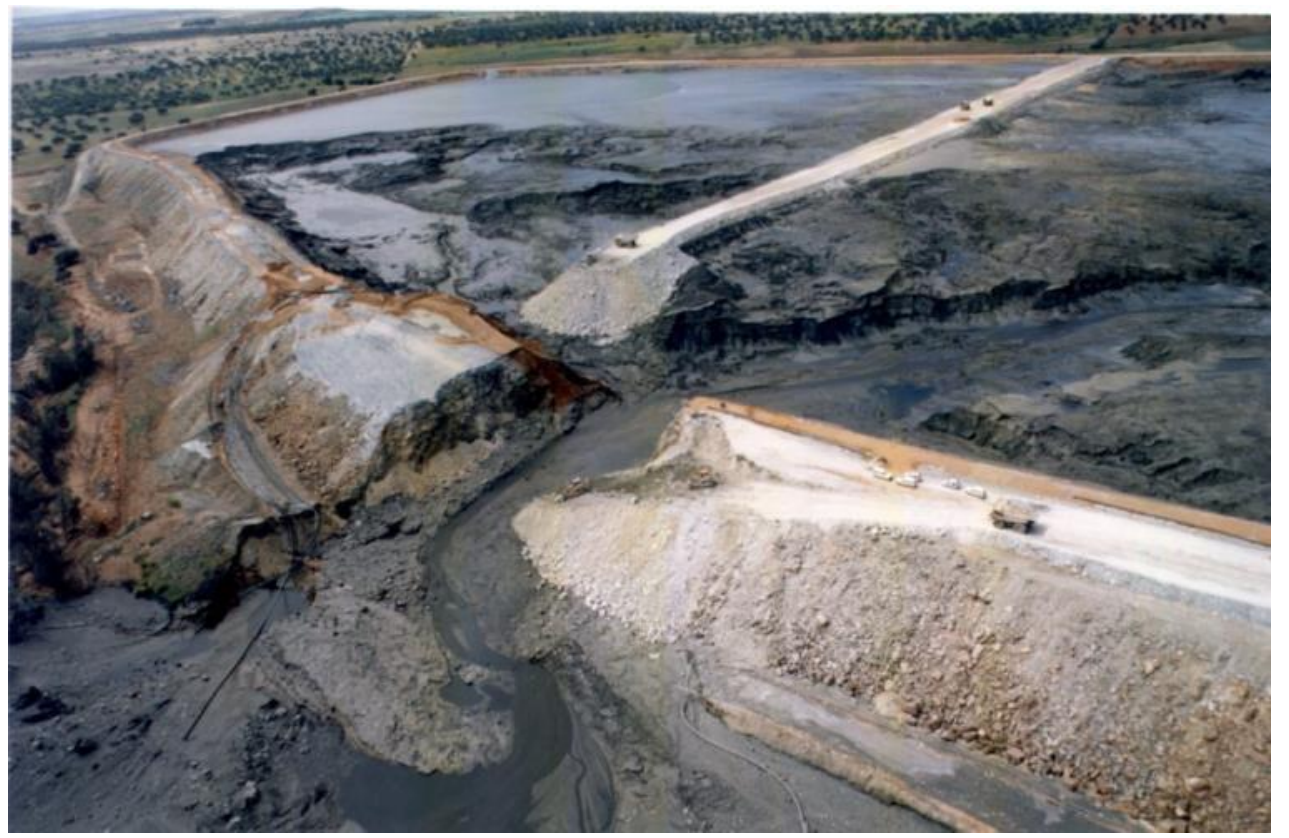

(c) $(1)$ 


\section{Materials: biochars}

pcampos@irnas.csic.es

Feedstock

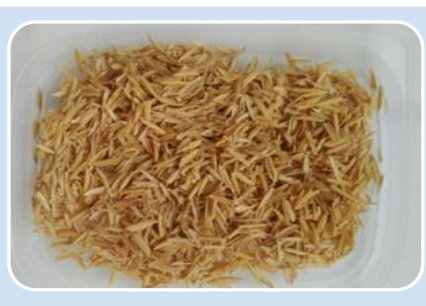

Rice Husk (RH)
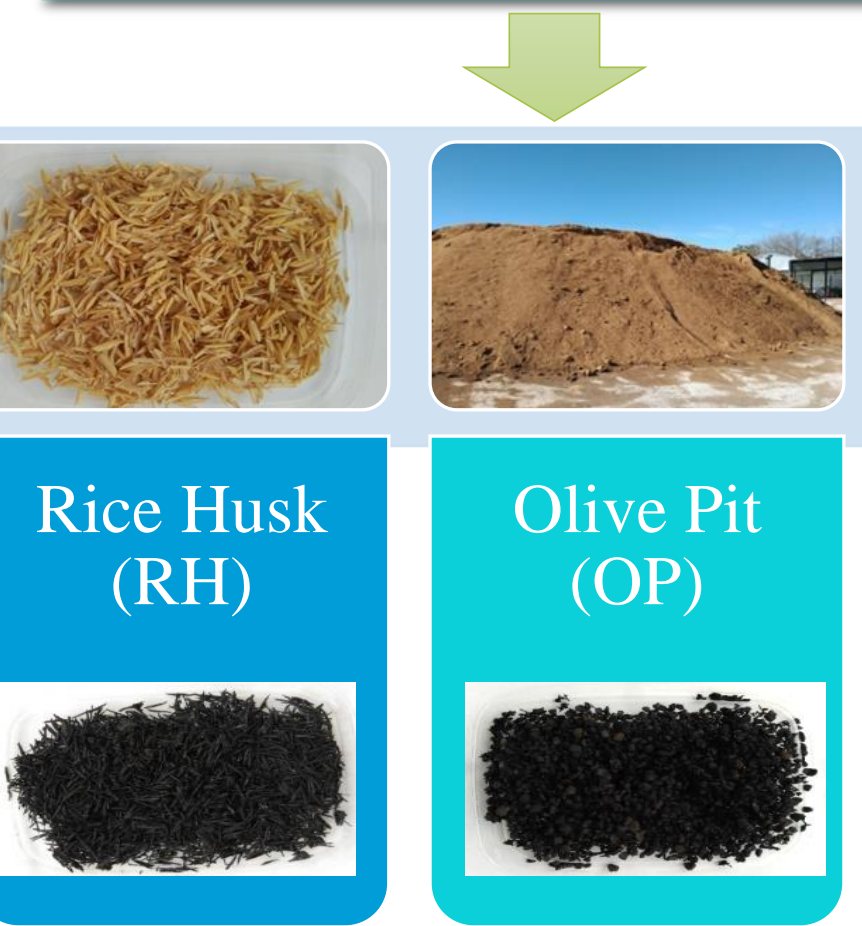

\section{Certified biochar (B1)}

- Material: Mixed wood sieving from wood chip

- Pyrolysis: $20 \mathrm{~min}, 620^{\circ} \mathrm{C}$

- Swiss Biochar, Laussane, Switzerland

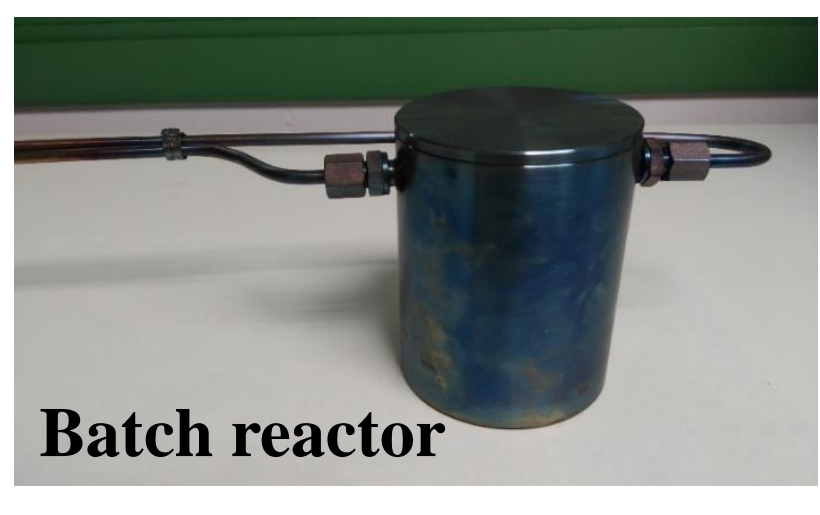
Biochar production
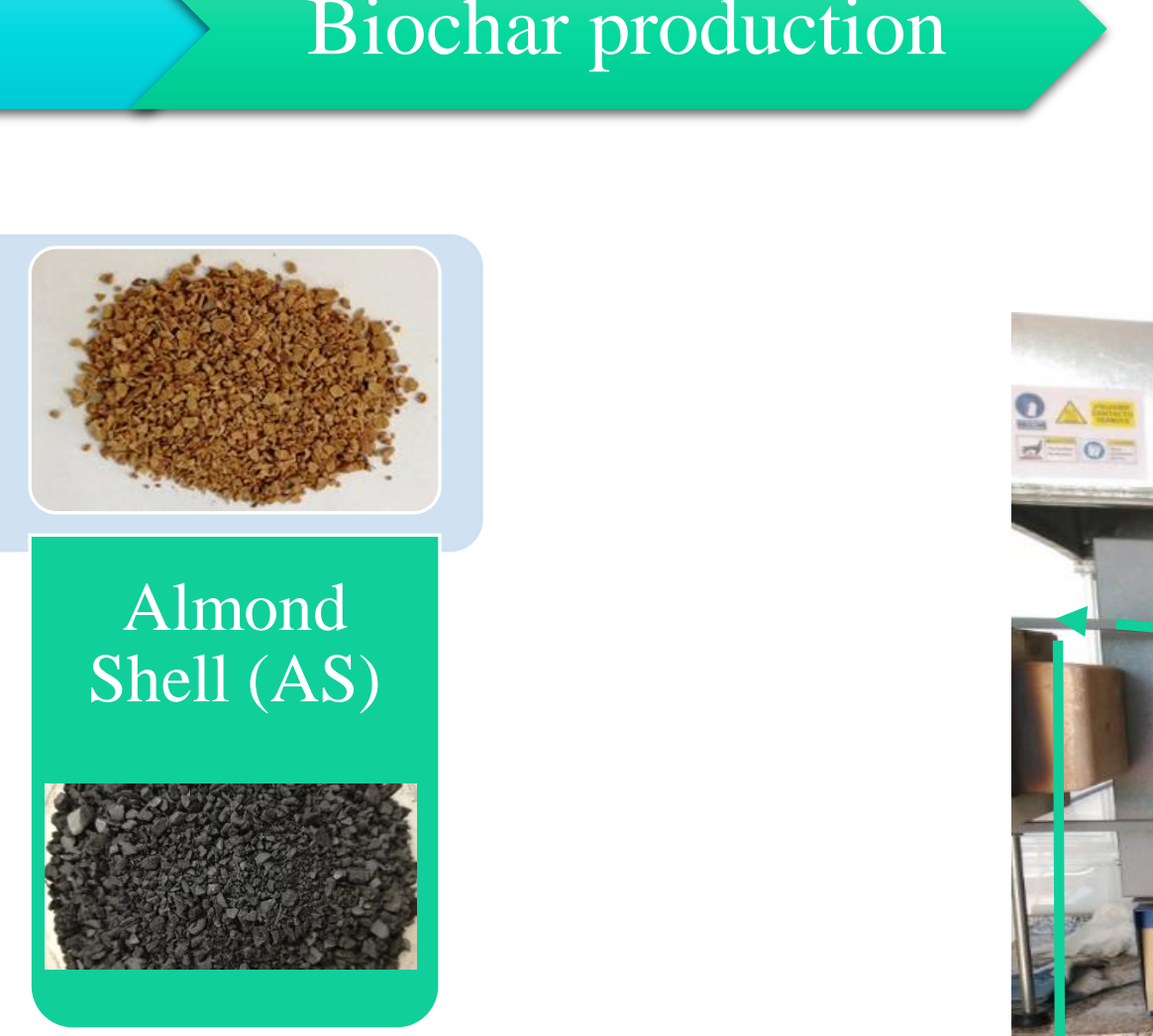

\section{Pyrolysis conditions}

- Temperature $500{ }^{\circ} \mathrm{C}$

- Time $2 \mathrm{~h}$

- $\mathrm{N}_{2}$ atmosphere

- Heating rate $20^{\circ} \mathrm{C} \mathrm{min}^{-1}$

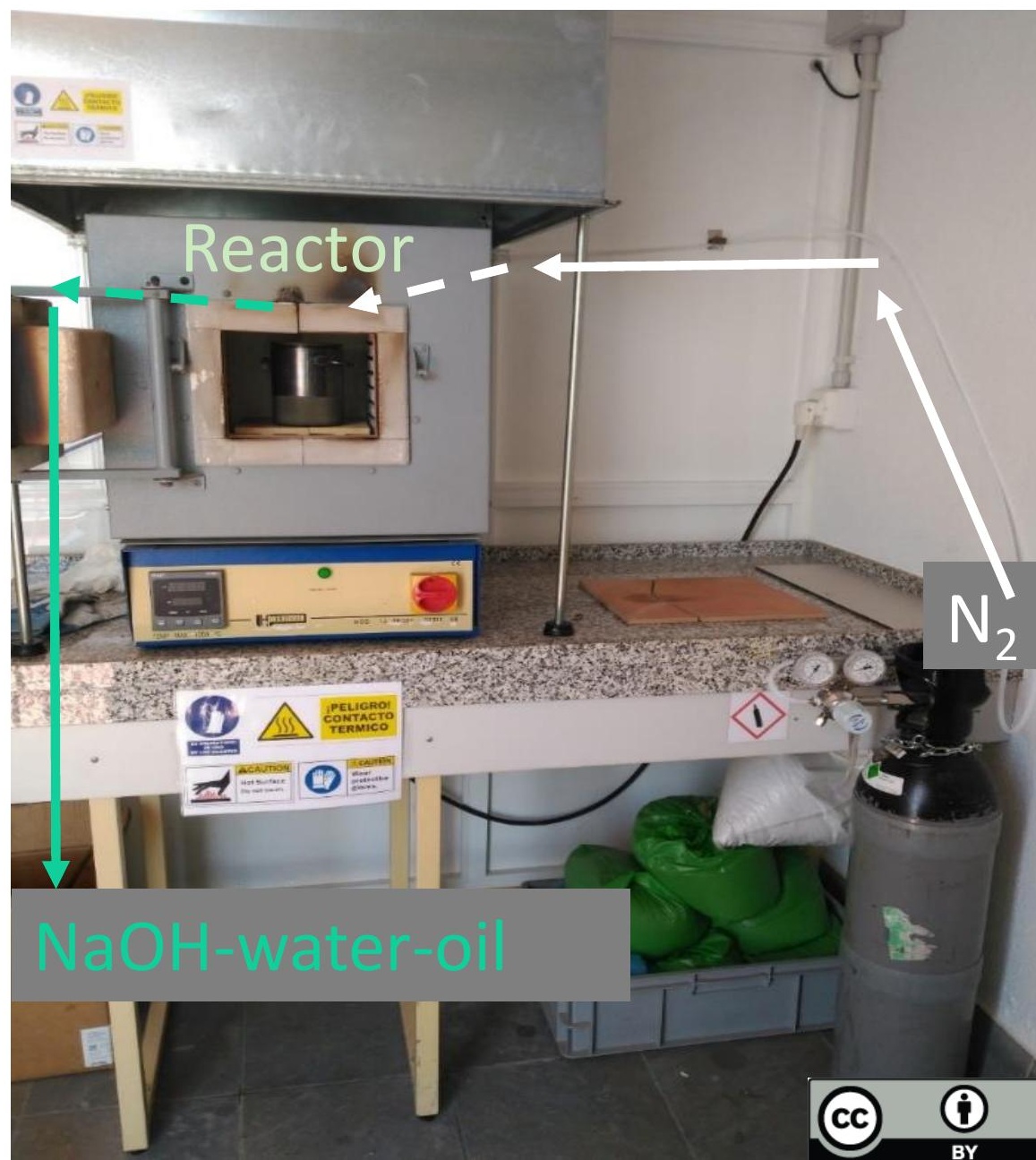




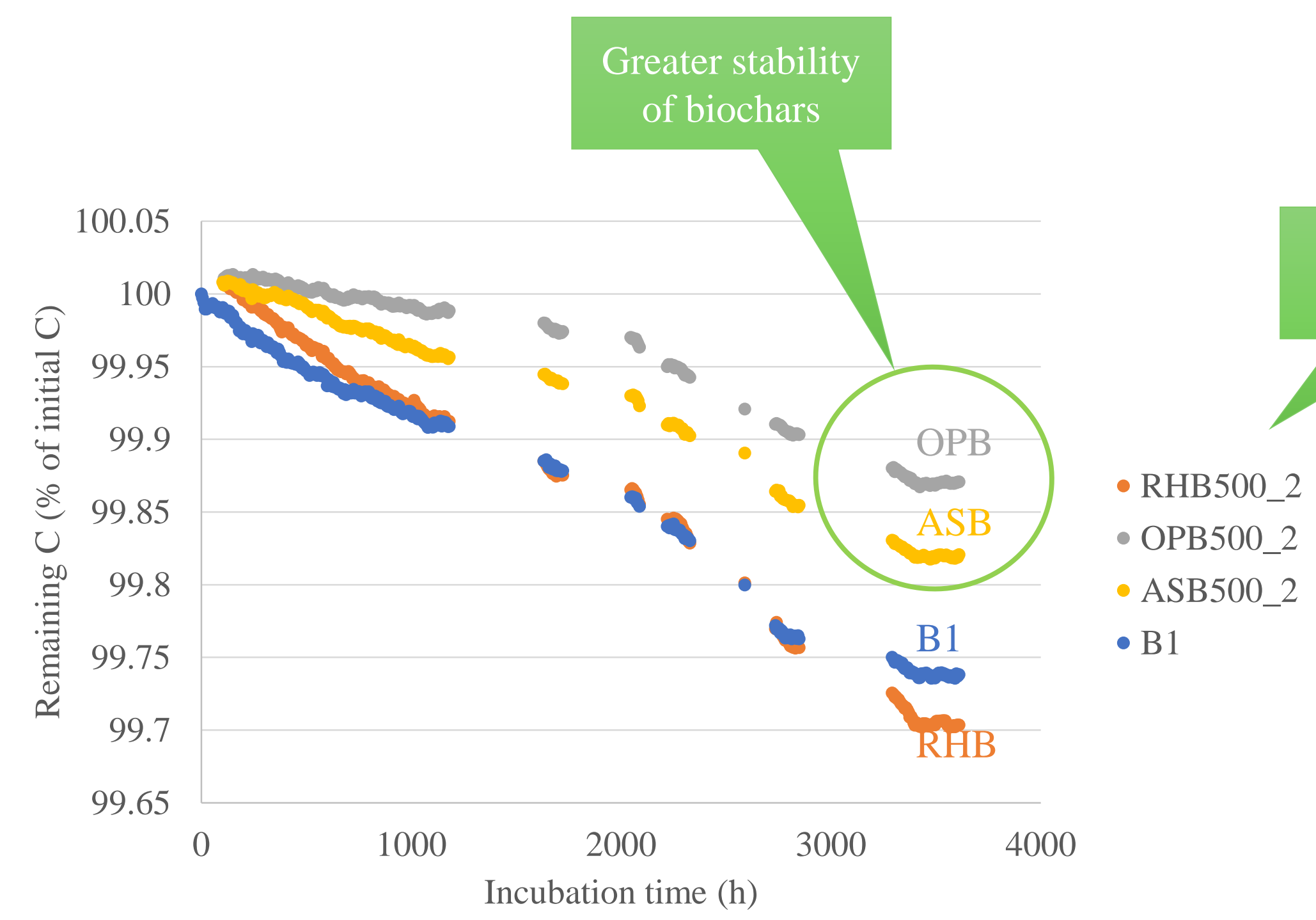

No fast pool

One-exponential model

$$
A=A_{2} \cdot \mathrm{e}^{-k_{2} t}
$$

Underestimation of biochar stability?

Leng et al. (2019), Sci. Total Environ. 664, 11-23

(c) (i) 


\section{cond}

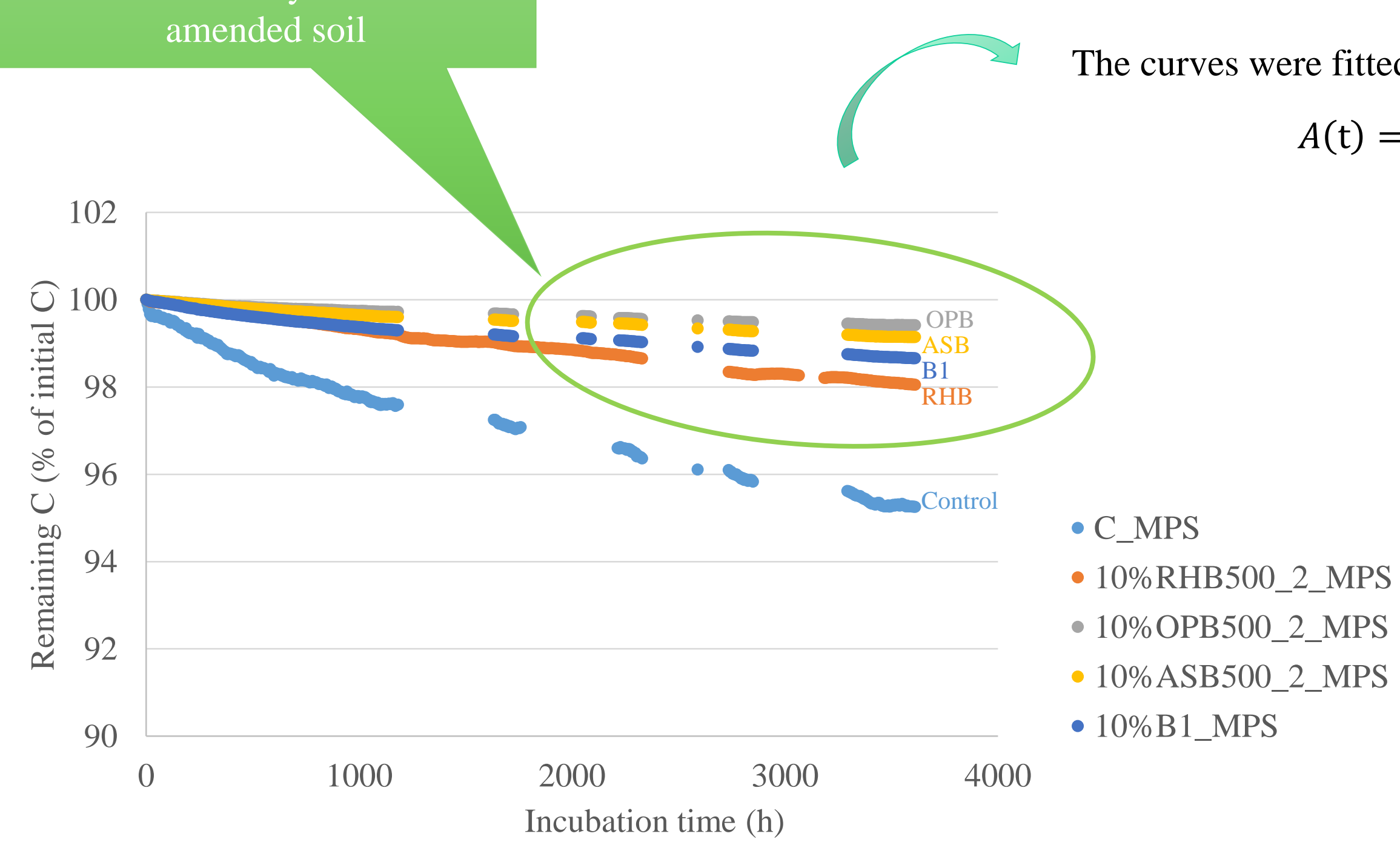

$$
A(\mathrm{t})=A_{1} \cdot \mathrm{e}^{-k_{1} t}+A_{2} \cdot \mathrm{e}^{-k_{2} t}
$$$$
\text { - C_MPS }
$$

-10\%RHB500_2_MPS

-10\%OPB500_2_MPS

10\%ASB500_2_MPS 


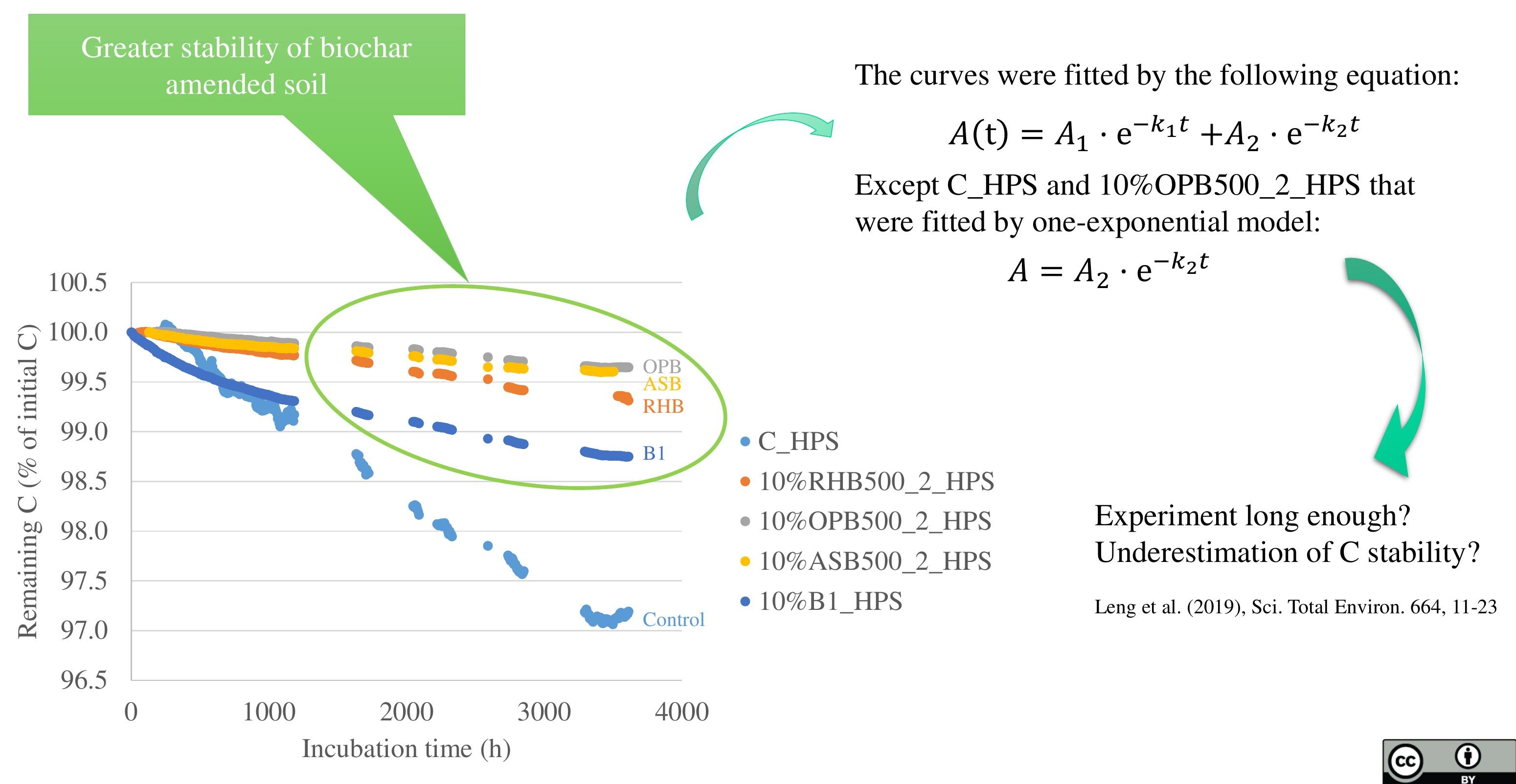




\section{Conclusions}

pcampos@irnas.csic.es

All biochars showed MRT > 100 years, being greater for OPB and ASB than RHB and the certified B1.

In moderately polluted soil, biochars:

- Did not modified the labile $\mathrm{C}$ fraction of soil.

- Increased $\mathrm{MRT}_{2}$ in 2-10 times in comparison to the control soil.

In highly polluted soil, biochars :

- Enhanced the degradation of a small fraction of labile $\mathrm{C}$.

- Increased in 2.8-13.1 times the $\mathrm{MRT}_{2}$ of the more recalcitrant $\mathrm{C}$ fraction of soil.

Longer experiments would be recommended in order to distinguish if the $\mathrm{C}$ fraction found in control HPS was the labile fraction or all $\mathrm{C}$ in this soil has this degradability. 


\section{Thank you for your attention}

Acknowledgements:

Grupo MOSS (IRNAS-CSIC)

MINEICO: CGL2016-76498-R and CGL2015-64811-P projects

Fundación Tatiana Pérez de Guzmán el Bueno 